Research Article

\title{
Study of the Effect of the Earthworm (Aporrectodea molleri) Cutaneous Excreta on Bacterial Growth
}

\author{
Lamia Yakkou (D), Sofia Houida (D), Mohammed Raouane $(\mathbb{D}$, Souad Amghar $(\mathbb{D}$, \\ and Abdellatif El Harti (DD
}

\begin{abstract}
Reserch Team, Lumbricidae, Improving Soil Productivity and Environment (LAPSE), Centre "Eau, Ressources Naturelles, Environnement et Développement Durable (CERNE2D), Mohammed V University in Rabat, Ecole Normale Supérieure (ENS), Avenue Med Belhassan El Ouazani, BP 5118, Takaddoum, Rabat, Morocco
\end{abstract}

Correspondence should be addressed to Lamia Yakkou; lamia_yakkou@um5.ac.ma

Received 17 January 2021; Accepted 21 May 2021; Published 29 May 2021

Academic Editor: Panos V. Petrakis

Copyright ( 2021 Lamia Yakkou et al. This is an open access article distributed under the Creative Commons Attribution License, which permits unrestricted use, distribution, and reproduction in any medium, provided the original work is properly cited.

The effect on the bacterial growth of Aporrectodea molleri cutaneous excreta has given very satisfactory results. The cutaneous excreta were obtained using the electrical stimulation technique, recently developed by our laboratory. This technique made it possible to collect the excreta in sufficient quantities while ruling out any possible interference with the chemical constituents of the soil. The effectiveness of cutaneous excreta, tested as culture media at different concentrations on bacterial growth, was justified not only by the high growth rates but also by the concentrations used, which were several times lower than those of the conventional medium (nutrient agar). Indeed, the maximum growth rate recorded by E. coli and P. fluorescens is, respectively, 1.32 times and 2.99 times greater than that observed on the conventional medium. These higher levels are obtained at optimal excretion concentrations which are, respectively, 8 times and 133.33 times lower than the concentration of the conventional medium. The efficacy interval that delimited the excretion concentrations tested covers 8 successive concentrations ranging from $0.0075 \%$ to $1 \%$ for $P$. fluorescens, while for E. coli, this interval covers only three concentrations ranging from $0.06 \%$ at $0.25 \%$. The growth rates of $P$. fluorescens are always higher than those of E. coli. The higher efficiency of excretions on the growth of $P$. fluorescens shows that this strain is more suited to use these excretions as a source of nutrients and to react positively to the stimulation of growth. These results, obtained in vitro, prove that, in the natural environment, the production of lubricating and viscous cutaneous excretions does not only have the role of facilitating the movement and digging of galleries by earthworms but also of enriching the soil in nutrients and growth factors with high added values for the activation and development of plant growth-promoting bacteria essential for soil fertility.

\section{Introduction}

Earthworms are the main animal biomass in the rhizosphere. According to the literature, these saprophagous animals constitute an enormous mass between 0.5 and 5 tonnes per hectare (fresh weight) which represents 60 to $80 \%$ of the animal biomass of the soil [1]. In this respect, they constitute the first terrestrial animal mass [2].

Earthworms dig thousands of $\mathrm{km}$ of galleries per hectare per year. These galleries constitute veritable aeration and drainage valves for the soil. Digging galleries in the ground cannot be possible without the presence of viscous cutaneous excreta (CEX), which protect earthworms against dehydration and facilitate movement $[3,4]$. Cutaneous excreta represent all fluids produced by the body surface of earthworms, which include the viscous mucus produced in the micropores of glandular cells lining the entire surface of the earthworm body, coelomic fluid released from the back coelomic pores, and urine excreted through the ventral urinary pores $[4,5]$ In addition, the cited fluids are known to be rich in nutrients such as nitrogen compounds and proteins [3].

The functioning of the terrestrial ecosystem is highly dependent on bacterial activity. In fact, soil bacteria are 
effectively involved in the decomposition and matter recycling processes. In agriculture, plant growth-promoting rhizobacteria (PGPR) promote the availability and uptake of nutrients by plants and limit the spread of pests $[6,7]$.

The interaction between bacteria and earthworms is essential for the natural improvement of soil fertility [8]. Furthermore, these burrowing animals are involved in the dynamics of the bacterial populations and ensure through their intestinal transit their dispersion in the soil $[9,10]$.

Therefore, given the large biomass of these invertebrate animals and the CEX produced continuously, our laboratory was interested in studying the impact of the CEX on bacterial growth, particularly on PGPR bacteria in the soil. In this work, we study the effect of the earthworm crude extract and CEX as culture media for the growth of E. coli, used here as a reference strain in the study of bacterial growth, and P. fluorescens as a PGPR strain characteristic of the soil.

\section{Materials and Methods}

2.1. Biological Material. Earthworms: Aporrectodea molleri is a kind of ubiquitous earthworm which is common to various ecosystems and agro-biocenoses in the western Mediterranean. This annelid was harvested in the region of Akrach about $20 \mathrm{~km}$ southeast of Rabat (Morocco).

Bacteria: the strain Escherichia coli ATCC 25922 was loaned to us by the National Institute of Hygiene (INH) of Rabat (Morocco). The strain Pseudomonas fluorescens belongs to the collection of our laboratory: Earthworm, Improving Soil Productivity and Environment (LAPSE) of Rabat (Morocco).

2.2. Preparation of Earthworm Cutaneous Excreta (CEX). The electrical stimulation technique, as developed by our laboratory [11], included the stages of prestimulation, stimulation sensu stricto, and poststimulation.

Prestimulation: this is the stage of fasting earthworms. This step is important because it avoids any interference of the cutaneous excreta with the contents of the earthworm digestive tract which is rejected through the anal and oral orifices during electrical stimulation. A fresh weight lot $\left(\mathrm{FW}_{\mathrm{EW} 1}=843.6 \mathrm{~g}\right)$ of Aporrectodea molleri was deprived of soil and food for 8-10 days. To prevent dehydration, the lot of earthworms was wrapped in a wet cotton tissue. Every two days, the moistened tissue was renewed, and then the whole was transferred to a second pot. The operation was repeated 4 to 5 times until the solid excrement was exhausted. By using a stereoscope, it was possible to make sure that the digestive tract of the earthworms has become almost empty. Finally, the lot of earthworms was rinsed quickly with distilled water. The fresh weight of the earthworm lot after the fasting period $\left(\mathrm{FW}_{\mathrm{EW} 2}\right)$ was determined.

Sensu stricto stimulation: the lot of earthworms from the fasting period was divided into 5 to 10 small lots depending on the volume of the excitation vessel (100 to $200 \mathrm{~mL}$ ) of the electrical stimulation device. The contraction of the earthworms promotes the massive production of cutaneous excreta. Each small lot of worms was bathed in 20 to $40 \mathrm{~mL}$ of distilled water depending on the volume of the excitation vessel. The electrodes connected to a double-pulse stimulator-type current generator were immersed in water in contact with the earthworms. Finally, each small lot was subjected to an electrical stimulation of a voltage $U=20 \mathrm{~V}$ with a frequency $F=100$ stimulations/second for a period of time $T=5$ minutes.

Poststimulation: at the end of each sensu stricto stimulation, each of the small lots was discharged into another container where it is rinsed with distilled water in order to recover the residue from the sticky cutaneous excreta that persist on the body of the worms. The small lots were then collected into a single lot and dried with Joseph paper. The final fresh weight of the lot after stimulation $\left(\mathrm{FW}_{\mathrm{EW} 3}\right)$ was determined. It was the difference between the initial fresh weight before stimulation $\left(\mathrm{FW}_{\mathrm{EW} 2}\right)$ and the final fresh weight after stimulation $\left(\mathrm{FW}_{\mathrm{EW}}\right)$ that determined the amount in the fresh weight of the cutaneous excreta produced $\left(\mathrm{FW}_{\mathrm{CEX}}\right)$. All the cutaneous excreta from the excitation containers as well as those from rinsing water were accumulated and then evaporated in an oven $\left(70^{\circ} \mathrm{C}\right)$ until the weight stabilizes. This was the final dry weight of cutaneous excreta $\left(\mathrm{DW}_{\mathrm{CEX}}\right)$ that will be used in the preparation of bacterial culture media.

2.3. Preparation of Bacterial Culture Media (CEX). A stock solution of $200 \mathrm{~mL}$ was prepared from $2 \mathrm{~g}$ of the dry residue of the excreta. From this solution, bacterial culture media were prepared by successive dilutions. $\mathrm{pH}$ was adjusted to neutral $\mathrm{pH}$. The $200 \mathrm{~mL}$ stock solutions were divided into 2 volumes of $100 \mathrm{~mL}$. The first volume $(100 \mathrm{~mL})$ constituted the $C^{\prime}{ }_{1}$ culture medium at $1 \%$. The second volume $(100 \mathrm{~mL})$ was made up to $200 \mathrm{~mL}$ with distilled water. After homogenization, this volume was divided into two; the first volume constituted the $C_{2}^{\prime}$ culture medium at $0.5 \%$, i.e., a dilution factor $(\mathrm{Fd})$ equal to $2 \mathrm{x}$. The second remaining volume $(100 \mathrm{~mL})$ was diluted in the same way as the above to obtain the $C_{3}^{\prime}$ medium at $0.25 \% ; \mathrm{Fd}=4 \mathrm{x}$. The series of successive dilutions continued until culture media $C^{\prime}{ }_{4}$ at $0.12 \%(\mathrm{Fd}=8 \mathrm{x}), C^{\prime}{ }_{5}$ at $0.0 .06 \%(\mathrm{Fd}=16 \mathrm{x}), C^{\prime}{ }_{6}$ at $0.0 .03 \%$ $(\mathrm{Fd}=32 \mathrm{x}), C^{\prime}{ }_{7}$ at $0.015 \%(\mathrm{Fd}=64 \mathrm{x})$, and $C_{8}^{\prime}$ at $0.0075 \%$ $(\mathrm{Fd}=128 \mathrm{x})$ were obtained.

The effect on the bacterial growth of culture media $\left(C_{1}, \ldots, C_{8}\right)$ based on cutaneous excreta (CEX) was compared to that of the conventional medium, nutrient agar (NA), which is composed of $2 \%$ of assimilable nutrients.

2.4. Inoculation and Growth Parameter. A 10x dilution series was carried out from the E. coli and P. fluorescens suspensions in order to obtain a number of colonies between 30 and 300 colonies/dish. From the dilution giving a comparable number of colonies, between 30 and $300,0.1 \mathrm{~mL}$ of suspension was deposited and spread at the centre of each prepared solid medium. The Petri dishes were finally incubated for 24 hours at $37^{\circ} \mathrm{C}$ for $E$. coli and at $30^{\circ} \mathrm{C}$ for $P$. fluorescens. Bacterial growth was determined by the average number of colonies formed per dish (NCF). 
2.5. Efficiency Parameter. The efficiency of the CEX ( $\left.\mathrm{E}_{\mathrm{CEX}}\right)$ was expressed compared to the conventional NA medium by two parameters.

Relative concentration $(\mathrm{RC})$ : it is the concentration of the CEX $\left(C_{\mathrm{CEX}}\right)$ relative to the concentration of the NA medium $\left(C_{\mathrm{NA}}\right): \mathrm{RC}=\left(C_{\mathrm{CEX}} / C_{\mathrm{NA}}\right) \times 100$.

Relative growth: it is the growth in the CEX media $\left(G_{\mathrm{CEX}}\right)$ compared to the growth in the conventional NA media $\left(G_{\mathrm{NA}}\right): \mathrm{RG}=\left(G_{\mathrm{CEX}} / G_{\mathrm{NA}}\right) \times 100$.

The CEX are said to be as effective or more effective than the conventional NA medium when $\mathrm{RC}$ is less than or equal to $100 \%$ (concentration of the extract is less than or equal to that of the NA medium) and RG is higher than or equal to $100 \%$ (growth in the extract is greater than or equal to that in the NA medium).

The CEX are described as ineffective when RC and RG do not meet the efficiency conditions mentioned above.

The optimal efficiency is designated when, at the concentration of the CEX, which is less than or equal to that of the NA medium, a maximum growth greater than or equal to that of the NA medium is obtained.

2.6. Analysis of Statistical Data. The data concerning the average number of colonies formed (NCF) were submitted to one-way ANOVA (SPSS software, version 22) in accordance with the experimental models. Significant differences were determined at a probability level of 0.05 , and the Tukey multiple comparison test was used to compare the means. All the data were expressed as means \pm standard error.

\section{Results}

3.1. Yield of Earthworm Cutaneous Excreta. A small amount of cutaneous excreta (CEX) was barely sufficient to perform biological tests planned on bacterial growth. The amount of CEX produced by electrical stimulation depends on the number of earthworms used. However, this large quantity of earthworms underwent a considerable reduction during the fasting period (prestimulation stage). A weight loss estimated at $40 \%$ during the fasting period (prestimulation stage) was observed. The weight loss estimated at $40 \%$ during this period is not only due to the drop in growth, observable by the decrease in the size of the earthworms and the disappearance of the clitellum in adult earthworms, but also by some cases of mortality.

The fresh weights recorded before and after the fasting period, as well as those obtained before and after the electrical stimulation, are presented in Tables 1 and 2. The total fresh and dry weights of the CEX released as well as the yield per $100 \mathrm{~g}$ of fresh weight of the earthworms are also shown.

The lot of earthworms used initially weighs $843.6 \mathrm{~g}$ $\left(\mathrm{FW}_{\mathrm{EW} 1}\right)$. This weight drops from $843.6 \mathrm{~g}\left(\mathrm{FW}_{\mathrm{EW1}}\right)$ to $506.16 \mathrm{~g}\left(\mathrm{FW}_{\mathrm{EW} 2}\right)$ after the fasting period (prestimulation stage). We find that only $60 \%$ of the fresh weight is actually used in the stage of sensu stricto stimulation. After stimulation, the fresh weight of the earthworms drops to $437.67 \mathrm{~g}$ $\left(\mathrm{FW}_{\mathrm{EW} 3}\right)$. The difference in the fresh weight between $\mathrm{FW}_{\mathrm{EW}}$ and $\mathrm{FW}_{\mathrm{EW} 3}$ indirectly gives the amount in fresh weight of
TABLE 1: Variations in fresh weight $\left(\mathrm{FW}_{\mathrm{EW} 1,2}\right)$ and yields before stimulation.

\begin{tabular}{lcc}
\hline Earthworm state & Fresh weight $(\mathrm{g})$ & Yield (\%) \\
\hline Before fasting & $\mathrm{FW}_{\mathrm{EW} 1}: 843.6$ & 100 \\
After fasting, before stimulation & $\mathrm{FW}_{\mathrm{EW} 2}: 506.16$ & 60 \\
\hline
\end{tabular}

The excreta yield is evaluated from the quantity of worms resulting from the prestimulation step $\left(\mathrm{FW}_{\mathrm{EW} 2}\right)$.

the CEX produced, $\mathrm{FW}_{\mathrm{CEX}}=68.5 \mathrm{~g}$, i.e., a yield of $13.5 \%$. This fresh yield is estimated in relation to $100 \mathrm{~g}$ of the fresh weight of the earthworms after the fasting period $\left(\mathrm{FW}_{\mathrm{EW} 2}\right)$. The CEX actually collected were reduced to dry residue at $70^{\circ} \mathrm{C}$. The dry weight of these excretions is $2.32 \mathrm{~g}\left(\mathrm{DW}_{\mathrm{CEX}}\right)$. The dry weight yield is estimated at $0.45 \%$ relative to $100 \mathrm{~g}$ of fresh weight of the worms resulting from the fasting period $\left(\mathrm{FW}_{\mathrm{EW} 2}\right)$.

\subsection{Effect of the Cutaneous Extract (CEX) on Bacterial Growth.} The effect of cutaneous excretion- (CEX-) based culture media at different $C_{n}$ concentrations $\left(C_{1}=1 \%, \ldots\right.$, $\left.C_{8}=0.0075 \%\right)$ on the growth of $E$. coli and P. fluorescens is shown, respectively, in Tables 3 and 4 . In both tables, we observe a variation in the number of colonies/dish (NCF) as a function of the concentration $\left(C_{n}\right)$ of excretions in the culture media. The extent of this variation depends on the bacterial strain tested.

Table 3 shows that, for the E. coli strain and at higher concentrations between $C_{1}=1 \%$ and $C_{4}=0.12 \%$, cutaneous excreta exert an inhibitory effect on growth. At lower concentrations between $C_{5}=0.06 \%$ and $C_{7}=0.0015 \%$, growth increases significantly $(p \leq 0.05)$ between $C_{6}$ and $C_{7}$. The maximum rate of NCF growth $=131.67$ to 132 colonies/ dish is recorded at these concentrations $\left(C_{6}\right.$ and $\left.C_{7}\right)$. At the concentration $C_{8}=0.00075 \%$, the growth is suddenly inhibited. The efficiency of the cutaneous excretions on the growth $\left(\mathrm{E}_{\mathrm{CEX} / \mathrm{NA}}\right)$ is evaluated compared to the conventional medium NA both by the relative growth (RG) which must be greater than or equal to $100 \%$ of that of the NA medium and the relative concentration (RC) which must be less than or equal to $100 \%$ of that of the NA medium.

The interval of efficiency of CEX meeting the conditions of effectiveness mentioned above is determined in bold in the table. In this interval between concentrations $C_{5}$ and $C_{7}$, the relative growth (RG) goes from $110.07 \%$ to $234.33 \%$. These values are greater than $100 \%$ of the relative growth of the NA medium. The relative concentration (RC) goes from $3 \%$ to $0.75 \%$ only compared to that of the NA medium which is assimilated to $100 \%$.

For the P. fluorescens strain (Table 4), growth starts at the concentration $C 1=1 \%$ with a rate of 70.67 colonies/dish which is already higher than that observed in the NA medium (67.7\% colonies/dish). Growth then intensifies as the CEX concentration decreases to reach a maximum level of $\mathrm{NCF}=299.67$ colonies $/$ dish at $C_{3}$ concentration $=0.25 \%$. The growth then slows down gradually between concentrations $C_{4}=0.12 \%$ and $C_{8}=0.0075 \%$. We note, however, that, in this range of low concentrations, the respective 
TABLE 2: Variations in fresh weight and yields during and after stimulation.

\begin{tabular}{lccc}
\hline Stimulation stage & Earthworm and cutaneous excreta state & Weight $(\mathrm{g})$ & Yield (\%) \\
\hline Sensu stricto stimulation stage & Earthworm after fasting, after stimulation & FW $_{\text {EW }}: 437.67$ & 86.46 \\
Poststimulation stage & Fresh cutaneous excreta & FW $_{\text {CEX }}: 68.49$ & 13.53 \\
& Dry cutaneous excreta & DW & 0.45 \\
\hline
\end{tabular}

The quantity of collected cutaneous excreta is evaluated in the fresh $\left(\mathrm{FW}_{\mathrm{CEX}}\right)$ and dry $\left(\mathrm{DW}_{\mathrm{CEX}}\right)$ state.

TABLE 3: Growth of Escherichia coli in solid media based on cutaneous excreta of earthworm Aporrectodea molleri (CEX) at different concentrations $\left(C_{n}: \mathrm{g} / 100 \mathrm{ml}\right)$.

\begin{tabular}{ccccccccccc}
\hline & \multicolumn{8}{c}{ Solid media based on cutaneous excreta of earthworm (CEX) at different concentrations $\left(C_{n}\right)$} \\
& & $C_{1}=1 \%$ & $C_{2}=0.5 \%$ & $C_{3}=0.25 \%$ & $C_{4}=0.12 \%$ & $C_{5}=0.06 \%$ & $C_{6}=0.03 \%$ & $C_{7}=0.015 \%$ & $C_{8}=0.0075 \%$ & NA \\
\hline \multicolumn{2}{c}{ NCF } & 0 & 0 & 0 & 0 & $62 \pm 7.21^{\mathrm{a}}$ & $131.67 \pm 9.27^{\mathrm{b}}$ & $\mathbf{1 3 2 . 0 0 \pm 1 0 . 9 6 ^ { \mathrm { b } }}$ & 0 & $56.33 \pm 2.02^{\mathrm{a}}$ \\
\hline \multirow{2}{*}{$\mathrm{E}_{\mathrm{CEX} / \mathrm{NA}}$} & RC (\%) & - & - & - & - & 3 & 1.5 & $\mathbf{0 . 7 5}$ & - & 100 \\
& RG (\%) & - & - & - & - & 110.06 & 233.75 & $\mathbf{2 3 4 . 3 3}$ & - & 100 \\
\hline
\end{tabular}

The growth is expressed by the average number ( \pm standard error) of colonies formed (NCF) at each concentration. The letters are significantly different at the confidence level of 0.05 between the culture media (degree of significance by the Tukey test $(p \leq 0.05)$ ). The effectiveness of the CEX extract ( $\mathrm{E}_{\mathrm{CEX} / \mathrm{NA}}$ ) is evaluated in relation to the conventional NA medium by, simultaneously, the relative concentration (RC) and the relative growth (RG).

growth rates are much higher than the growth rate of the NA medium. The zone of effectiveness of cutaneous excreta on the growth of $P$. fluorescens includes the entire range of concentrations tested, ranging from $C_{1}=1 \%$ to $C_{8}=0.0075 \%$. In this zone, the values of the relative growth are greater than $100 \%$ of the NA medium. These values range from $104.38 \%$ to $340.22 \%$. Also, the values of the relative concentration of cutaneous excreta are always less than $100 \%$ of NA. These values are between $50 \%$ and $0.39 \%$ only. Optimal efficiency is obtained at the $C_{3}$ concentration $=0.25 \%$ where growth is greatest $(\mathrm{NCF}=299.67)$. At this concentration, the relative growth is $442.64 \%$ with respect to NA. On the contrary, the relative concentration is only $12.5 \%$ compared to NA.

Figure 1 illustrates the parallel evolution of the relative growth (RG) and the relative concentration (RC) as a function of the increasing concentration of CEX in the zones of effectiveness on the growth of E. coli and P. fluorescens. For comparison, the $\mathrm{RG}$ and $\mathrm{RC}$ values of the conventional medium NA, which are set at $100 \%$, are also shown. In this figure, the zone of efficiency on the growth of $E$. coli comprises only three concentrations $\left(C_{7}, C_{6}\right.$, and $\left.C_{5}\right)$, whereas in $P$. fluorescens, this zone covers all the excretion concentrations $\left(C_{8}\right.$ to $\left.C_{1}\right)$. In both zones, the relative growth for the two strains is always greater than $100 \%$ of the NA medium. Conversely, the relative CEX concentration, identical for the two strains, is always less than $100 \%$ of the NA medium. We note that the lower the relative CEX concentration, the greater the relative growth of the two strains.

In the growth efficiency zone for E. coli, the maximum relative growth of $234.33 \%$ is 2.34 times greater than that of $100 \%$ of the NA medium. In the area of efficiency for P. fluorescens, the maximum relative growth of $442.64 \%$ is 4.42 times greater than that of $100 \%$ of the NA medium. The comparison of the values of the maximum relative growth of the two strains shows that $P$. fluorescens has a value which is 1.88 times higher.
The actual optimal CEX concentrations between $C_{6}=0.03 \%$ and $C_{7}=0.015 \%$, which gave the best growth rate for E. coli, correspond to optimal relative concentrations of $1.5 \%$ to $0.75 \%$ only. These values are 66.66 times to 133.33 times less than $100 \%$ of the NA medium. Likewise, the actual optimal concentration of $C_{3}=0.25 \%$, which gave the best growth rate for $P$. fluorescens, corresponds to an optimal relative concentration of only $12.5 \%$ which is 8 times less than $100 \%$ of the NA medium.

\section{Discussion}

Since earthworms represent the first terrestrial animal mass and taking into account the quantity of cutaneous excreta produced permanently during the activity period of these animals, our laboratory was interested in the study of the impact of the earthworm crude extract and earthworm cutaneous excreta in the rhizosphere, more particularly on the microbial activity essential for soil fertility. The paucity of studies relating to the in vitro effect is understandable by the difficulty of collecting these cutaneous excretions in sufficient quantity for the performance of repetitive biological tests. The originality of our work is that it was carried out entirely in vitro outside the soil, eliminating possible interference with the biological and chemical components of the soil. In addition, the earthworm fasting period eliminated the risk of interference of digestive tract contents. This study could not have been carried out without the technique of electrical stimulation of the production of skin excretions, developed by our laboratory [11]. This technique allowed the collection of cutaneous excreta in sufficient quantity and in a very short time. The amount of excreta collected in this work was $13.53 \mathrm{~g}$ wet weight and $0.45 \mathrm{~g}$ dry weight. This amount was adjusted from $100 \mathrm{~g}$ fresh weight of fasting earthworms.

In this work, the effectiveness of CEX on bacterial growth was determined by comparison with a conventional culture medium (nutrient agar) of known composition and concentration. We were able to affirm that the crude extracts of the cutaneous excreta (CEX) have considerable potential 


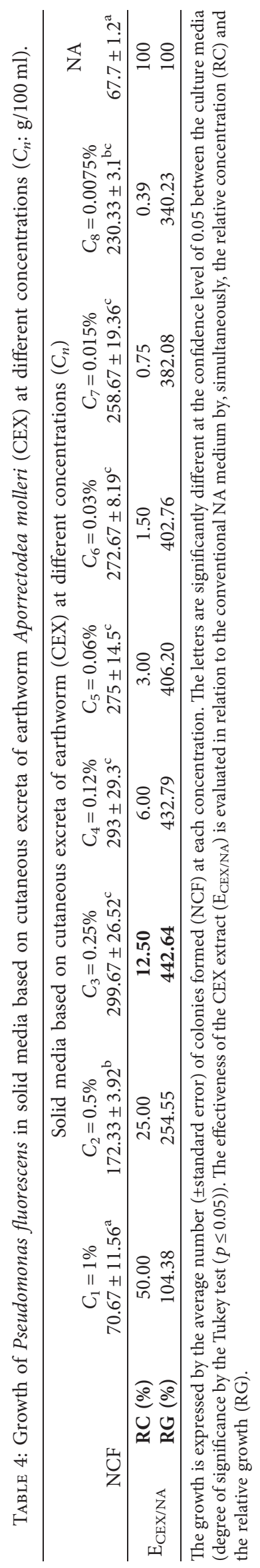




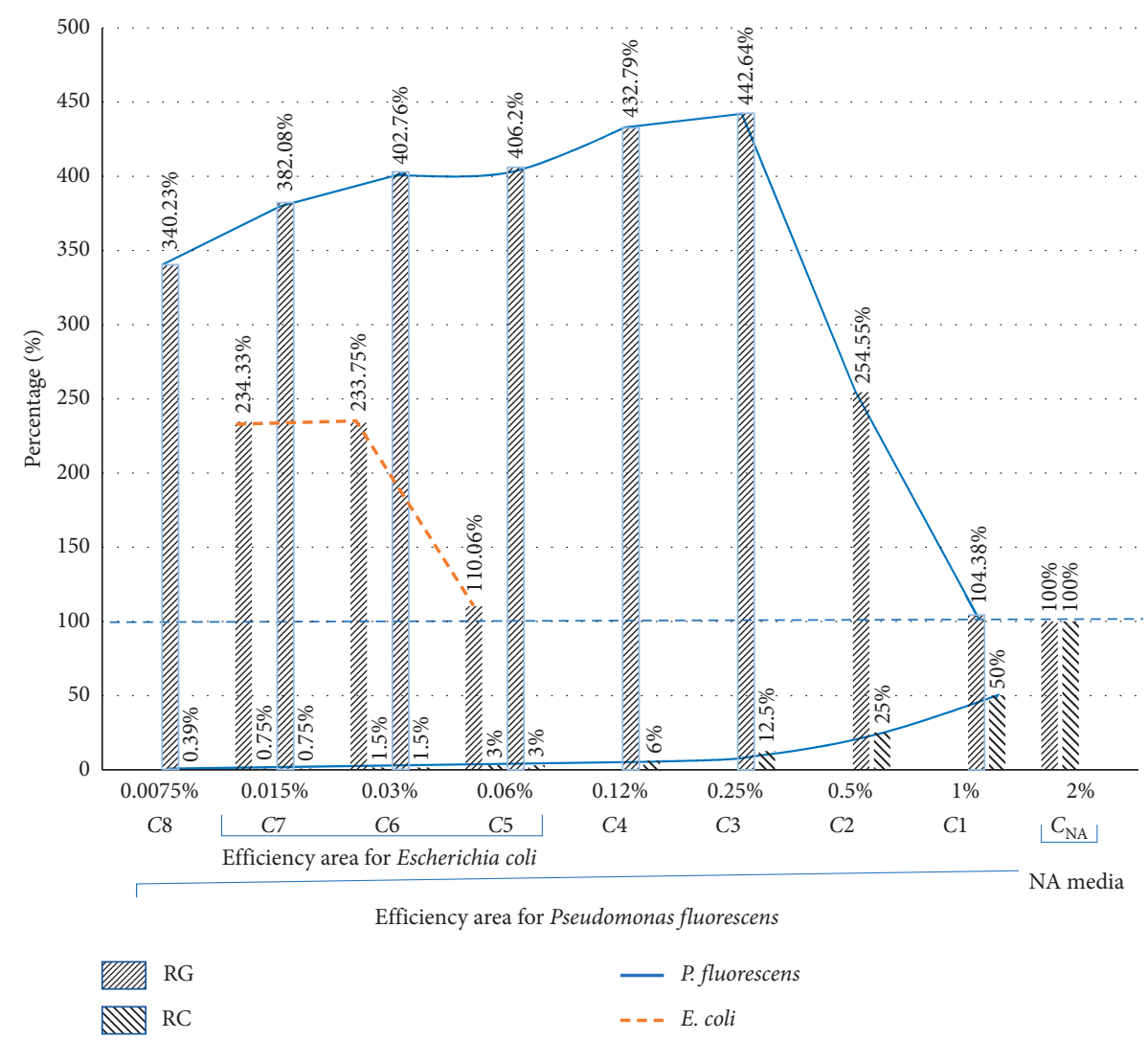

FIGURE 1: Evolution of the relative growth (RG) of Escherichia coli and Pseudomonas fluorescens and the relative concentration (RC) of cutaneous excreta (CEX) in the areas of efficiencies. The relative growth and concentration are expressed as a percentage (\%) relative to those of the conventional NA medium which represent $100 \%$. $C_{\mathrm{NA}}$ : concentration of the NA medium at $2 \%$.

for bacterial growth at much lower concentrations compared to the conventional NA medium. Moreover, knowing that the average fresh weight of an adult specimen of Aporrectodea molleri is approximately $1 \mathrm{~g}$, we can therefore express the effect of the fresh weight of CEX produced by one earthworm (fresh weight). Indeed, the concentration $C_{1}=1 \mathrm{~g}$ dry weight of CEX per $100 \mathrm{~mL}$ corresponds to, approximately, 29.52 grams of fresh weight of CEX per $218.16 \mathrm{~g}$ fresh weight of earthworms, which equals $13.5^{*} 10^{-2}$ grams of fresh weight of CEX produced by one earthworm. The media based on CEX would therefore be more diversified in nutrients and growth substances.

Based on the results concerning the effect of cutaneous excreta (CEX) on bacterial growth, it can be said that CEX of Aporrectodea molleri are very favourable for bacterial growth. The efficiency on growth is demonstrated not only by the high growth rates compared to the conventional NA medium but also by concentrations of CEX used which are much lower. Thus, in the natural system, CEX of Aporrectodea molleri, released in small amounts but in a continual manner, could be a source of nutrients and growth stimulants for PGPR bacteria such as P. fluorescens.

The interesting results, obtained in vitro in this work, are in agreement with those observed directly in the natural system. Indeed, it has been reported that "mucus," which refers to cutaneous excreta, has a priming effect on the stimulation of microbial activity and the decomposition of plant residues in the soil [12]. According to Bityutskii et al. [12], "mucus" promotes humification by increasing humic substances by $20 \%$ to $30 \%$. Oleynik and Byzov [3] demonstrated that earthworm cutaneous excreta affect the formation of soil microbial communities by stimulation. According to Huang and Xia [13], "mucus" positively stimulates the growth of proteobacteria but negatively affects firmicutes during decomposition. This selective action is believed to be the cause of the proteins' abundance in the vermicomposting system, where earthworms are used to convert organic waste into fertilizer [14]. Moreover, CEX increase the dehydrogenase activity by up to $20.7 \%$ [13]. This result could suggest, according to Huang and Xia [13], that the "mucus" provides the favourable conditions for microbial growth. Furthermore, the concentrations of nitrogen and dissolved phosphorus showed higher values in the "mucus" $[13,15]$ The very positive results obtained by our laboratory and those cited in the literature testify to the richness and diversity of CEX in nutrients and growth factors that can be assimilated by microorganisms. According to Cortez and Bouché [16], "mucus" of earthworms is made up of $69 \%$ proteins and peptides and $31 \%$ carbohydrates. In addition, the coelomic fluid and urine which constitute the cutaneous excreta indicate the presence of several molecules such as glycogen, ammonia, urea, uric acid, and allantoin and several types of proteins [17]. 


\section{Conclusion}

While all species of earthworms are animated by permanent movements in the rhizosphere, these movements are accompanied by the continual production of cutaneous excreta. Thus, due to the richness and diversity of their composition, cutaneous excreta would continually supply the soil with nutrients and growth factors, promoting the development of PGPR bacteria essential for soil fertility.

\section{Data Availability}

The data used to support the findings of this study are included within the article.

\section{Conflicts of Interest}

The authors declare that there are no conflicts of interest regarding the publication of this paper.

\section{Acknowledgments}

This work was carried out with the support and the grant of the National Center for Scientific and Technical Research (CNRST) within the framework of the Research Excellence Scholarship Program.

\section{References}

[1] M. Bouché, "Les vers de terre," Recherche, vol. 156, pp. 796804, 1984.

[2] C. A. Edwards and J. R. Lofty, "Effects of earthworm inoculation upon the root growth of direct drilled cereals," The Journal of Applied Ecology, vol. 17, no. 3, p. 533, 1980.

[3] A. S. Oleynik and B. A. Byzov, "Response of bacteria to earthworm surface excreta," Mikrobiologiya, vol. 77, pp. 854-862, 2008.

[4] S. Salmon, "Earthworm excreta (mucus and urine) affect the distribution of springtails in forest soils," Biology and Fertility of Soils, vol. 34, no. 5, pp. 304-310, 2001.

[5] M. Santocki, A. Falniowski, and B. Plytycz, "Restoration of experimentally depleted coelomocytes in juvenile and adult composting earthworms Eisenia andrei, E. fetida and Dendrobaen aveneta," Applied Soil Ecology, vol. 104, pp. 163-173, 2016.

[6] J. M. Raaijmakers, T. C. Paulitz, C. Steinberg, C. Alabouvette, and Y. Moënne-Loccoz, "The rhizosphere: a playground and battlefield for soilborne pathogens and beneficial microorganisms," Plant and Soil, vol. 321, no. 1-2, pp. 341-361, 2009.

[7] G. Sellan, J. Thompson, N. Majalap, R. Robert, and F. Q. Brearley, "Impact of soil nitrogen availability and $\mathrm{pH}$ on tropical heath forest organic matter decomposition and decomposer activity," Pedobiologia, vol. 80, p. 150645, 2020.

[8] F. Wu, J. H. C. Wan, S. Wu, and M. Wong, "Effects of earthworms and plant growth-promoting rhizobacteria (PGPR) on availability of nitrogen, phosphorus, and potassium in soil," Journal of Plant Nutrition and Soil Science, vol. 175, no. 3, pp. 423-433, 2012.

[9] M. Aira, M. Pérez-losada, and J. Domínguez, "Diversity, structure and sources of bacterial communities in earthworm cocoons," Scientific Reports, vol. 8, pp. 1-9, 2018.

[10] M. C. Picón, E. S. Teisaire, M. S. Zutara, and S. A. Giunta, "Identification of the intestinal microbial community of eisenia andrei (annelida: lumbricidae) raised in different substrates," Munis Entomology \& Zoology Journal, vol. 10, pp. 101-106, 2015.

[11] M. Raouane and A. El Harti, "Effect of electrical stimulation on the production of cutaneous excreta by the earthworm Lumbricus terrestris L," Bulletin de la Société Royale des Sciences de Liège, vol. 89, 2020.

[12] N. P. Bityutskii, E. I. Maiorov, and N. E. Orlova, "The priming effects induced by earthworm mucus on mineralization and humification of plant residues," European Journal of Soil Biology, vol. 50, pp. 1-6, 2012.

[13] K. Huang and H. Xia, "Role of earthworms' mucus in vermicomposting system: biodegradation tests based on humification and microbial activity," Science of the Total Environment, vol. 610-611, pp. 703-708, 2018.

[14] X. Fu, G. Cui, K. Huang et al., "Earthworms facilitate the stabilization of pelletized dewatered sludge through shaping microbial biomass and activity and community," Environmental Science and Pollution Research, vol. 23, no. 5, pp. 4522-4530, 2016.

[15] G. G. Brown, I. Barois, and P. Lavelle, "Regulation of soil organic matter dynamics and microbial activity in the drilosphere and the role of interactions with other edaphic functional domains," European Journal of Soil Biology, vol. 36, 2000.

[16] J. Cortez and M. B. Bouché, "Composition chimique du mucus cutané de Allolobophora chaetophora chaetophora (Oligo-chaeta: lumbricidae)," Comptes Rendus de l'Académie des Sciences III, vol. 305, pp. 207-210, 1987.

[17] C. A. Edwards and C. J. Bohlen, Biology and Ecology of Earthworms, Chapman \& Hall, London, UK, 3rd edition, 1996. 